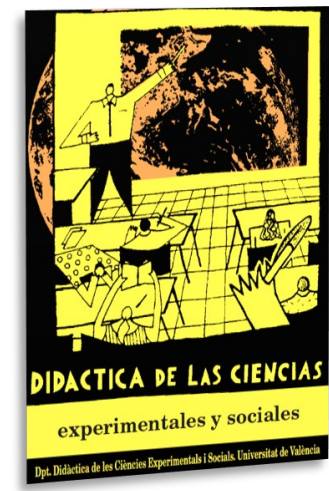

\title{
La actitud hacia el bienestar animal de estudiantes universitarios y personas mayores de sesenta años: el género $y$ la edad como elementos clave
}

\author{
Attitudes towards animal welfare among \\ university students and people over sixty: \\ gender and age as key factors
}

DOI: $10.7203 / D C E S .40 .18048$

\begin{abstract}
Juan Gabriel Reyes-Jiménez
Asociación Medioambiental Educando por la Naturaleza: EDUCANATURA, juangral@hotmail.com ORCID iD: https://orcid.org/0000-0002-0392-8491
\end{abstract}

Jerónimo Torres-Porras

Universidad de Córdoba, jeronimo.torres@uco.es

ORCID iD: https://orcid.org/0000-0003-1900-7870

Jorge Alcántara-Manzanares

Universidad de Córdoba, b62almaj@uco.es

ORCID iD: https://orcid.org/0000-0003-2482-1615

Juan Rafael Rodríguez-Hidalgo

Asociación Medioambiental Educando por la Naturaleza: EDUCANATURA, ciencia2050@hotmail.com

ORCID iD: https://orcid.org/0000-0003-4623-2301

\begin{abstract}
RESUMEN: El objetivo de este trabajo es evaluar y comparar la actitud hacia el bienestar animal de estudiantes de los Grados de Educación Primaria e Infantil y de personas mayores de sesenta años jubiladas pertenecientes a Centros de Participación Activa, a través del cuestionario AWA (Animal Welfare Attitude), siendo la primera vez que se estudia la actitud de este último grupo. Los resultados han mostrado que existen diferencias significativas en la actitud hacia el bienestar animal en función de los grupos de pertenencia señalados y del género. La actitud recogida ha sido favorable en general, pero la más favorable es la del grupo de estudiantes, lo que resulta positivo teniendo en cuenta que en un futuro cercano tendrá la responsabilidad de ejercer su profesión en las aulas.
\end{abstract}

Palabras ClaVe: bienestar animal, profesorado en formación, personas jubiladas, género, actitudes.

ABSTRACT: The objective of this work is to evaluate and compare attitudes towards animal welfare of students of the Primary and Early Childhood Education Degrees and of retired people over the age of sixty, belonging to Active Participation Centers, through the AWA (Animal Welfare Attitude) questionnaire, being the first time that attitudes of this last group has been studied. The results have shown that there are significant differences in attitudes towards animal welfare depending on the indicated groups and gender. Attitudes were favorable in general, but the most favorable were those of the student group, which is positive considering that in the near future they will have the responsibility of exercising their profession in the classrooms.

KEYWORDS: animal welfare, pre-service teachers, retired persons, gender, attitudes.

Fecha de recepción: julio de 2020 Fecha de aceptación: mayo de 2021

Agradecemos a la dirección, al profesorado y a los usuarios de los Centros de Participación Activa, así como al alumnado universitario su participación en este proyecto. 


\section{INTRODUCCIÓN}

El bienestar animal es cada vez más importante desde los puntos de vista social, político, ético y científico (Velde, Aarts y Van Woerkum, 2002). A lo largo de la historia, las relaciones entre seres humanos y animales han sido muy variadas, yendo desde la utilización de estos como medio de trabajo, como alimentos, como entretenimiento, como protector o como símbolo de culto, hasta su utilización para la investigación, la ayuda a personas con alguna discapacidad o incluso para cubrir el componente afectivo (Gutiérrez, Granados y Piar, 2007; Serpell, 2000). Pero es en el último siglo, donde han comenzado a tener relevancia estas relaciones, apareciendo los movimientos en defensa de los animales (López, 2005).

El concepto clásico de bienestar animal comenzó a definirse hasta concretarse cinco libertades definitorias del estado de bienestar de un animal, las cuales son: libre de hambre y sed, libre de molestias, libre de dolor, libre para expresar los comportamientos naturales de su especie y libre de miedo o estrés (Brambell, 1965; Webster, 2001), aunque luego se han ido especificando más necesidades ligadas al bienestar, especialmente garantizar que los animales tengan mayores oportunidades de experimentar estados de bienestar positivos (McCulloch, 2013; Mellor, 2016). En la sociedad actual, el concepto de bienestar animal está adquiriendo más repercusión, lo que justifica la promoción de la educación y concienciación hacia los animales, inculcando en las personas actitudes favorables hacia estos. Una actitud es la posición de una persona sobre una dimensión bipolar evaluativa o afectiva con respecto de un objeto, acción o evento y representa el sentimiento favorable o desfavorable de una persona hacia algún objeto estímulo (Ajzen y Fishbein, 1977). Cuando se miden actitudes se miden actitudes hacia algo (Vallejo, 2006).

Una consecuencia de la mayor repercusión del concepto de bienestar animal son las numerosas críticas por parte de varios sectores de la sociedad que ha recibido la producción ganadera intensiva, que han motivado importantes cambios legislativos sobre bienestar animal en Europa (Horgan y Gavinelli, 2006; Lundmark, Berg, Schmid, Behdadi y Röcklinsberg, 2014; María, 2006). Por otra parte, por su influencia determinante en el bienestar animal, ha proliferado la psicología animal como un aspecto a investigar (Mendl, Burman, Parker y Paul, 2009). Estos cambios han motivado un debate social, con partidarios de un carácter más utilitario hacia los animales (instrumentos de trabajo, entretenimiento, alimento), ignorando sus condiciones de vida y matanza, y por otro lado un sector poblacional cada vez más extenso, consciente del concepto de bienestar animal e implicado con el mismo (Gutiérrez, Granados y Piar, 2007). Cabe señalar que es con el aumento de la población urbana, cuando crece la preocupación por los animales y el medioambiente, por el desarrollo cada vez más numeroso de relaciones afectivas hacia los animales de compañía, extendiéndose la preocupación por el sufrimiento animal y reclamando una legislación que lo regule y garantice el bienestar de estos (Dawkins, 2004; Herzog y Golden, 2009). En España por ejemplo, se propuso en 2017 una reforma legislativa, pero ha sido en abril de 2021 cuando se ha aprobado en el Congreso de los Diputados la tramitación de una proposición de ley en la que los animales dejarán de ser considerados cosas para pasar a ser seres vivos con sensibilidad. Diferentes estudios evidencian que cuanto mayor es el conocimiento de las necesidades fisiológicas y la etología de los animales, mayor es la capacidad de identificar el sufrimiento animal y, por tanto, mayor disposición para la protección de los animales (Melik, 2007).

El término maltrato animal hace referencia a un amplio abanico de comportamientos violentos sobre los animales por parte de los seres humanos, que pueden derivar en consecuencias negativas sobre su salud física y psíquica, llegando en casos extremos a causar la muerte del animal (Fernández-Álvarez, 2019). Según el artículo 337 de la Ley Orgánica del Código Penal, el maltrato animal supone un delito, donde se impone una pena de prisión de tres meses a un año a quien, por cualquier medio o procedimiento, maltrate injustificadamente a un animal doméstico o amansado, causándole la muerte o lesiones que menoscaben gravemente su salud. Uno de los principales factores determinantes del maltrato animal por placer es la falta de empatía con el animal, agravada 
por la existencia de intencionalidad de causarle daño (Ascione, 2015). Bentham fue uno de los primeros pensadores que reivindicó la legislación de espectáculos de ocio con animales (Bentham, 1789; en Mosterín, 1998, p.219). Actualmente, muchas de estas prácticas violentas hacia los animales siguen existiendo en distintos países: peleas de animales, caza, tauromaquia, circos...

En los últimos años está habiendo una mayor tendencia a relacionar el bienestar de los animales como señal de calidad del producto alimenticio que proporcionan, considerando mejores alimentos aquellos que proceden de animales que gozan de mayor bienestar durante su vida (Miranda-de la Lama et al., 2013). Se convierte pues en una alternativa frente a la producción ganadera en explotaciones intensivas, el tipo de ganadería cuyo objetivo prioritario es la obtención de alimentos de calidad de manera compatible con el bienestar animal, la salud pública y el medioambiente, mediante técnicas y procedimientos modernizados (García Menacho y García Romero, 2012).

Por todo ello, los estudios sobre actitudes hacia los animales, desde los relacionados con el manejo de animales de granja, su transporte y sacrificio, hasta los más generales han proliferado (Fernie, Tribe, Murray, Lisle y Phillips, 2012; George, Slagle, Wilson, Moeller y Bruskotter, 2016; Prokop y Randler, 2018; Spooner, Schuppli y Fraser, 2012, 2014).

Existe documentación en publicaciones de autores como Broida (1993), Herzog, Betchart y Pittman (1991), Hills (1995), Kellert (1988), Mathews y Herzog (1997), Paul y Serpell (1993) y Phillips et al. (2012) que determinan diferentes factores que afectan a las actitudes hacia los animales, destacando entre estos la personalidad de los individuos, el género y su orientación sexual, sus creencias religiosas, su ideología política y ética, la propiedad de animales de compañía, así como otras variables demográficas como la edad y la raza o país.

La importancia que ha adquirido el bienestar animal implica la necesidad de estudiar las actitudes de la población desde el punto de vista de la educación, ya que la educación ambiental cada vez está más presente en los currículos debido a la necesidad de modificar actitudes y comportamientos que están provocando serios problemas ambientales.

En general, la educación se considera de vital importancia para formar actitudes (Ajzen, 2001; Ajzen y Fishbein, 2000). Según Jamieson et al. (2012) se debe fomentar en educación la mejora de la consideración hacia los animales y su cuidado, de tal manera que exista una responsabilidad social e individual. En la Comunicación de la Comisión al Parlamento Europeo, al Consejo y al Comité Económico y Social Europeo relativa a la estrategia de la Unión Europea para la protección y el bienestar de los animales 2012-2015 (COM 2012-2015, p. 12), se muestra la importancia de la educación para motivar el auge del bienestar animal como un aspecto indispensable en la vida actual.

Son varias las escalas que han sido elaboradas para conocer los aspectos relacionados con las actitudes hacia el bienestar animal, como el uso de animales, su integridad o experimentación animal (Hills, 1995; Henry, 2004; Herzog, Betchart, y Pittman, 1991; Phillips et al., 2011, 2012); sin embargo, los estudios realizados en España sobre las actitudes hacia el bienestar animal han estado centrados en estudiantes de distintas edades y no se han tenido en cuenta las personas mayores ya jubiladas, cuyas actitudes también son importantes y que dependiendo de dichas actitudes se podrían diseñar estrategias educativas para trabajar con este sector de la sociedad (Mazas, Fernández Manzanal, Zarza y María, 2013; Mazas y Fernández-Manzanal, 2019a, 2019b; Menor-Campos, Hawkins y Williams, 2019; Menor-Campos et al., 2019). Por lo que se pretende en este trabajo es analizar y confrontar las actitudes de dos grupos, estudiantes universitarios y personas jubiladas mediante la escala AWA (Animal Welfare Attitude Scale) de Mazas et al. (2013).

Aparentemente ambos grupos poblacionales son muy distales, personas mayores que fueron educados en un posicionamiento más pragmático en cuanto a la relación del ser humano con el resto de los animales, y personas jóvenes que se están formando para ser educadoras y, por tanto, a priori, 
más cercanas a la educación para la defensa del bienestar animal. Podríamos esperar entonces una actitud más favorable del grupo de jóvenes respecto al de jubilados (María, 2006).

\section{OBJetivos}

El objetivo principal es evaluar y comparar la actitud hacia el bienestar animal de estudiantes de los Grados de Educación Primaria e Infantil y de personas mayores de sesenta años jubiladas a través de una escala ya validada de interés para conocer y establecer las relaciones con las diferentes variables sociodemográficas.

Se pretende a su vez comprobar las posibles diferencias en la actitud hacia el bienestar animal en función de las variables sociodemográficas: grupos de pertenencia (universitarios y personas mayores de sesenta años) y género (masculino y femenino), así como si otras variables pueden estar influyendo en estos grupos, como la localidad de residencia o la tenencia de mascotas. Esto permitiría mostrar la posible necesidad de abordar desde la educación el fomento de actitudes más favorables hacia el bienestar animal, tanto en los grados de educación, como en los centros de capacitación activa o en las aulas universitarias para mayores.

\section{Metodología}

Esta investigación se desarrolla mediante un diseño no experimental de carácter descriptivo. En él se describen las diferentes actitudes recopiladas a través de unos cuestionarios realizados sin manipulación de variable alguna (Bisquerra, 2000).

\subsection{Descripción de la muestra de personas participantes}

En el estudio han participado un total de 306 individuos de los cuales 303 cumplimentaron el cuestionario completamente, realizándolo a lo largo del año 2019, incluyéndose dentro de este periodo los cursos académicos 2018/2019 y 2019/2020.

Por tanto, la muestra que forma parte del estudio quedó compuesta por 303 individuos, de los cuales 201 son estudiantes universitarios de la Facultad de Ciencias de la Educación de de la Universidad de Córdoba, siendo 105 pertenecieron al Grado de Educación Infantil y 96 al Grado de Educación Primaria y los otros 102 participantes son personas mayores de sesenta años jubiladas pertenecientes a los Centros de Participación Activa de las poblaciones de Castro del Río, Espejo y de Córdoba capital.

Las razones por las que se eligió esta muestra fueron, entre otras, la importancia de conocer las actitudes del alumnado de la Facultad de Ciencias de la Educación, por su proyección profesional, ya que ello se va a reflejar, en cierto modo, en su labor educativa respecto al bienestar animal y los aspectos medioambientales, tan importantes en la educación de este siglo que se enfrenta a graves problemáticas medioambientales, entre las que se encuentran la pérdida de biodiversidad y donde la educación ambiental posee un papel fundamental, ya que entre sus objetivos se encuentra el cambio de actitudes y comportamientos para un futuro más sostenible y respetuoso con el medio ambiente y con el resto de seres vivos. Y, por otro lado, la importancia de conocer la perspectiva de las personas mayores de sesenta años jubiladas, ya que es un grupo escasamente estudiado y puede aportar datos de gran interés al tratarse de unas actitudes basadas en su mayor experiencia vivencial y a unas relaciones con los animales con un alto componente utilitario, debido sus condicionantes culturales y que tienen además la posibilidad de seguir formándose en los Centros de Capacitación Activa, por lo que es necesario establecer su actitud hacia el bienestar animal, si se quieren diseñar estrategias educativas.

La muestra puede dividirse atendiendo a diferentes variables de estudio, una de las variables sociodemográficas analizada ha sido el grupo de pertenencia, en el que hemos diferenciado los dos 
grandes grupos estudiados, por un lado, el estudiantado universitario y por otro las personas jubiladas, así contamos con un 66,4\% de estudiantes universitarios y un 33,6\% de personas mayores de 60 años jubiladas. Otra variable es el género, para el que cabe resaltar que el número de individuos de género masculino del grupo de los universitarios del Grado de Educación Infantil es tan reducido que se ha decidido unir dicho grupo con el de los universitarios del Grado de Educación Primaria, dado las grandes similitudes entre grupos. De total de los sujetos participantes en el estudio hay una predominancia del género femenino con un $74 \%$, respecto al género masculino que está formado por un $26 \%$. El $52 \%$ de la muestra son mujeres estudiantes universitarias, el 15\% de la muestra son estudiantes universitarios hombres, el $22 \%$ del total de la muestra, mujeres mayores de sesenta años jubiladas y el 11\% de la muestra son hombres jubilados.

\subsection{Instrumentos y técnicas de recogida de información}

Para realizar la investigación se ha utilizado el cuestionario AWA (Animal Welfare Attitude), basado en una escala Likert de 5 puntos, ya validada por Mazas et al. (2013). Esta escala tiene en cuenta la definición de actitud como una predisposición aprendida para responder a un objeto de una manera consistentemente favorable o desfavorable, teniendo las siguientes dimensiones claramente diferenciadas: la cognitiva, la afectiva y la conductual (Ajzen, 2005).

Los 29 ítems de la escala se encuentran agrupados en cuatro ámbitos o componentes (tabla 1): el maltrato de los animales por placer o por desconocimiento (Componente 1- C1), el empleo de animales para el ocio o maltrato por uso en espectáculos u otras formas de divertimento con animales (Componente 2- C2), las situaciones en las que se hallan los animales de granja (Componente 3- C3) o el abandono de animales, concretamente mascotas (Componente 4- C4). La escala completa la denominaremos C-Total. Al cuestionario se le han añadido algunas variables sociodemográficas como: grupo (universitarios/jubilados), género (femenino/masculino), residencia (pueblo/ciudad) y tenencia de mascota (sí/no).

TABLA 1. Cuestionario utilizado agrupado por componentes

\begin{tabular}{cl}
\hline$N^{0}$ ítem & \multicolumn{1}{c}{ Componente y enunciado } \\
\hline & C1: Maltrato animal por placer o por desconocimiento \\
$\mathbf{1}$ & Los animales sufren, se lastiman cuando los golpeas \\
$\mathbf{3}$ & Cada animal doméstico debe ser cuidado. \\
$\mathbf{5}$ & Golpo derecho a golpear a un animal si me molesta. \\
$\mathbf{6}$ & A veces me divierto persiguiendo animales. \\
$\mathbf{1 0}$ & Nunca le pegaría a mi mascota para educarla. \\
$\mathbf{1 4}$ & Destruir los lugares de vida de los animales no importa; ellos pueden encontrar otro. \\
$\mathbf{2 0}$ & Matar animales pequeños, por ejemplo, gorriones o palomas, es mi afición. \\
& C2: Maltrato animal por ocio y divertimento \\
$\mathbf{4}$ & Me gustan las corridas de toros porque es un signo de identidad española. \\
$\mathbf{8}$ & Los animales utilizados en espectáculos no son respetados. \\
$\mathbf{1 2}$ & Me preocupa que los toros sufran en la plaza de toros, aunque solo dure unos minutos. \\
$\mathbf{1 5}$ & Los espectáculos con animales son eventos donde la gente se divierte a costa de su \\
& sufrimiento. \\
$\mathbf{1 8}$ & Los toros son animales valientes, su objetivo es morir en las plazas. \\
$\mathbf{2 5}$ & Creo que eventos sociales como las corridas de toros no deberían existir en una sociedad \\
$\mathbf{2 8}$ & civilizada. \\
& Me siento muy triste cuando los toros sufren en la plaza de toros mientras la gente se divierte. \\
$\mathbf{7}$ & Creo que los animales padecen enfermedades tanto físicas como psicológicas.
\end{tabular}


9 Suelo comer huevos de gallinas criadas en jaulas.

13 Los animales de granja deben mantenerse en jaulas para que puedan ser manejados fácilmente.

17 Los animales de granja no se ven afectados por sus condiciones de vida porque son seres vivos inferiores.

22 Los animales de granja no sufren.

29 Los animales agresivos deben ser sacrificados inmediatamente porque no pueden ser domesticados.

\section{C4: Maltrato animal por abandono}

16 Dejaría un animal en el campo si me aburriera de él.

19 Los animales abandonados se sienten libres.

21 Abandonas animales es una práctica muy cobarde e irresponsable.

23 Me encantaría colaborar con un refugio para animales abandonados.

24 Nunca abandonaría a mi mascota.

26 Los animales deben estar protegidos por la ley.

27 Siempre compro mascotas en tiendas de mascotas; los de los refugios de animales son viejos y feos.

Fuente: Mazas et al., 2013

\subsection{Análisis estadísticos}

Para estos análisis se han utilizado los programas estadísticos STATISTICA 8.0 (Statsoft Inc., Tulsa, Oklahoma, USA), SPSS 15.0 (SPSS, Chicago, IL, U.S.A.), así como Excel de Microsoft.

Se realizó un análisis de frecuencia K-S (Kolmogorov-Smirnov) para medir la normalidad del total de la muestra Actitud general o C-Total; $(\mathrm{K}-\mathrm{S} \mathrm{d}=0,16 ; \mathrm{p}<0,01)$ y de las cuatro dimensiones del estudio: $\mathrm{C} 1$ (K-S d=0,16; $\mathrm{p}<0,01), \mathrm{C} 2(\mathrm{~K}-\mathrm{S} \mathrm{d}=0,10 ; \mathrm{p}<0,01), \mathrm{C} 3(\mathrm{~K}-\mathrm{S} \mathrm{d}=0,12 ; \mathrm{p}<0,01)$ y $\mathrm{C} 4(\mathrm{~K}-\mathrm{S}$ $\mathrm{d}=0,17 ; \mathrm{p}<0,01)$. Los resultados obtenidos en todas las dimensiones muestran que los datos no siguen una distribución normal, por lo que se han aplicado pruebas no paramétricas en el análisis de las respuestas del cuestionario.

Por otro lado, para conocer la fiabilidad de los datos obtenidos con la escala AWA, se ha utilizado el coeficiente de fiabilidad $\alpha$ de Cronbach. En la tabla 2 podemos observar los resultados obtenidos en el presente estudio, adjuntando además los obtenidos por Mazas et al. (2013) para poder hacer una comparativa de ambos estudios. Además, Mazas et al. (2013) mostraba los valores de Kaiser-Meyer-Olkin (KMO) para examinar el grado de correlación entre variables y obtenía un valor de 0,87 (valores superiores a 0,8 se consideran aceptables, Kaiser, 1974), en este estudio obtenemos un valor de 0,83 que también se considera aceptable.

TABLA 2. Resultados análisis de fiabilidad con Coeficiente $\alpha$ de Cronbach

\begin{tabular}{lcc}
\hline \multicolumn{1}{c}{ Dimensiones } & $\begin{array}{c}\text { Resultados del presente } \\
\text { estudio }\end{array}$ & $\begin{array}{c}\text { Resultados del estudio } \\
\text { de Mazas et al. (2013) }\end{array}$ \\
\hline C-Total: Actitud general & 0,86 & 0,89 \\
$\begin{array}{l}\text { C1: Maltrato animal por placer o } \\
\text { por desconocimiento }\end{array}$ & 0,51 & 0,79 \\
C2: Ocio con animales & 0,83 & 0,83 \\
C3: Animales de granja & 0,47 & 0,76 \\
C4: Abandono de animales & 0,67 & 0,68 \\
\hline
\end{tabular}

Fuente: elaboración propia 
Como puede comprobarse en la tabla, el $\alpha$ de Cronbach de actitud general (C-Total) es elevado, lo que muestra la fiabilidad de la escala. Sin embargo, dos de las dimensiones (C1 y C3) poseen un $\alpha$ de Cronbach reducido. La prueba mostraba que eliminando en $\mathrm{C} 1$ el ítem $10 \mathrm{y}$ en $\mathrm{C} 3$ el ítem 7, mejoraría el valor del $\alpha$ de Cronbach, sin embargo, se ha decidido no eliminarlos para utilizar el mismo cuestionario validado por Mazas et al. (2013) y poder comparar con esos grupos de estudiantes de instituto y universitarios. Aunque posteriormente en Mazas y Fernández Manzanal (2018b) se eliminan 4 ítems $(8,9,14,25)$, pero no coinciden con los que indicaba nuestro análisis, por lo que como se ha comentado, se decidió utilizar el cuestionario completo.

Por otra parte, algunos autores sugieren que es necesario calcular el coeficiente omega (Ventura-León y Caycho-Rodríguez, 2017), ya que aporta mayor fiabilidad; el resultado de este coeficiente es de 0,88 lo que puede considerarse aceptable al estar entre 0,7 y 0,9 (Campo-Arias y Oviedo, 2008).

Para determinar qué variables sociodemográficas de las estudiadas son las determinantes de la actitud hacia el bienestar animal, se ha utilizado el test de Kruskal Wallis, prueba no paramétrica que nos permite comparar entre varios grupos independientes estableciendo rangos y cuya interpretación simplificada puede realizarse utilizando sus medianas. Además, se han realizado comparaciones intra-grupo mediante el test U de Mann-Whitney.

\section{RESULTADOS}

La edad, condicionante principal del grupo de pertenencia (universitarios y mayores de sesenta años jubilados) y el género han sido las variables determinantes de los cuatro grupos de estudio que se han utilizado en el análisis como factores o componentes finales que definieron la actitud hacia el bienestar animal.

Los datos extraídos de las puntuaciones medias de los cuatro grupos de estudio, obtenidos de las combinaciones de las variables grupo de pertenencia y género, determinan una mayor valoración hacia el bienestar animal del género femenino perteneciente al grupo de los universitarios (tabla 3). En el otro extremo están los participantes en el estudio de género masculino y del grupo de los mayores de sesenta años jubilados, que muestran los resultados más bajos. El orden de los componentes según sus medias en los 4 grupos estudiados es similar: $\mathrm{C} 1>\mathrm{C} 4>\mathrm{C} 3>\mathrm{C} 2$.

TABLA 3. Puntuación media (y medianas) de actitud hacia el bienestar animal por grupo y género

\begin{tabular}{lrrrr}
\hline \multicolumn{1}{c}{ Dimensiones } & Universitarias & Universitarios & Jubiladas & Jubilados \\
\hline C-Total: Actitud General & $4,43(4,48)$ & $4,11(4,17)$ & $3,83(3,82)$ & $3,59(3,55)$ \\
C1: Maltrato animal por placer & $4,70(4,75)$ & $4,42(4,50)$ & $4,36(4,37)$ & $4,09(4,06)$ \\
o por desconocimiento & & & & \\
C2: Ocio con animales & $4,15(4,37)$ & $3,67(3,62)$ & $3,42(3,31)$ & $3,02(3,00)$ \\
C3: Animales de granja & $4,24(4,33)$ & $4,00(4,16)$ & $3,50(3,66)$ & $3,48(3,41)$ \\
C4: Abandono de animales & $4,61(4,71)$ & $4,34(4,42)$ & $3,97(3,92)$ & $3,76(3,92)$ \\
\hline
\end{tabular}

Fuente: elaboración propia

La aplicación de la prueba no paramétrica de Kruskal Wallis para los distintos grupos para la actitud general ante el bienestar y para cada uno de los componentes (tabla 4) muestra diferencias significativas en todos los análisis (C-Total: $\mathrm{H}(3, \mathrm{~N}=303)=97,38 ; \mathrm{p}<0,001 ; \mathrm{C} 1: \mathrm{H}(3, \mathrm{~N}=303)$ $=56,55 ; \mathrm{p}<0,001 ; \mathrm{C} 2: \mathrm{H}(3, \mathrm{~N}=303)=60,58 ; \mathrm{p}<0,001 ; \mathrm{C} 3: \mathrm{H}(3, \mathrm{~N}=303)=63,87 ; \mathrm{p}<0,001 ; \mathrm{C} 4: \mathrm{H}$ $(3, \mathrm{~N}=03)=63,46 ; \mathrm{p}<0,001)$. 
Puede verse como el grupo más independiente, que se presenta como más diferente al resto, es el de las universitarias. El siguiente grupo que muestra más resultados significativos de independencia es el de los jubilados. Ambos son los polos opuestos respecto a su actitud hacia el bienestar animal. Por su parte, no se muestran como independientes entre sí los grupos jubiladas y jubilados. Sin embargo, cuando se comparan ambos con el grupo universitarios, se puede comprobar cómo éste es más similar al grupo jubiladas que al grupo jubilados. Así, universitarios y jubiladas solo se muestran independientes en relación con las respuestas de C-Total y del Componente $\mathrm{C} 3$, relativo a la relación con los animales de granja. Mientras que universitarios y jubilados se muestran significativamente diferentes en cuanto a la actitud hacia el bienestar animal en todos los componentes menos en $\mathrm{C} 1$. En cualquier caso, la escala AWA general o C-Total muestra diferencias entre todos los grupos menos entre jubiladas y jubilados.

TABLA 4. Resultados de comparación de grupos de las pruebas de Kruskal-Wallis. En negrita los resultados que muestran diferencias significativas $(\mathrm{p}<0,05)$

\begin{tabular}{|c|c|c|c|c|c|}
\hline & & Universitarias & Universitarios & Jubiladas & Jubilados \\
\hline \multirow[t]{4}{*}{ C-Total } & Universitarias & & $\mathrm{Z}=3,32 ; p=0,005$ & $Z=7,79 ; \mathbf{p}<0,001$ & $Z=7,68 ; p<0,001$ \\
\hline & Universitarios & $\mathrm{Z}=3,32 ; p=0,005$ & & $Z=2,96 ; P=0,017$ & $\mathrm{Z}=3,92 ; p<0,001$ \\
\hline & Jubiladas & $Z=7,79 ; p<0,001$ & $Z=2,96 ; p=0,017$ & & $Z=1,53 ; p=0,7$ \\
\hline & Jubilados & $\mathrm{Z}=7,68 ; \mathrm{p}<0,001$ & $\mathrm{Z}=3,92 ; \mathbf{p}<\mathbf{0 , 0 0 1}$ & $\mathrm{Z}=1,53 ; \mathrm{p}=0,7$ & \\
\hline \multirow[t]{4}{*}{$\mathrm{C} 1$} & Universitarias & & $Z=3,61 ; p=0,001$ & $\mathrm{Z}=5,43 ; \mathbf{p}<0,001$ & $Z=5,94 ; p<0,001$ \\
\hline & Universitarios & $\mathrm{Z}=\mathbf{3 , 6 1 ; p = 0 , 0 0 1}$ & & $\mathrm{Z}=0,92 ; \mathrm{p}=1$ & $\mathrm{Z}=2,26 ; \mathrm{p}=0,14$ \\
\hline & Jubiladas & $\mathrm{Z}=5, \mathbf{4 3} ; \mathbf{p}<0,001$ & $\mathrm{Z}=0,92 ; \mathrm{p}=1$ & & $Z=1,59 ; p=0,65$ \\
\hline & Jubilados & $Z=5,94 ; p<0,001$ & $Z=2,26 ; p=0,14$ & $Z=1,59 ; p=0,65$ & \\
\hline \multirow[t]{4}{*}{$\mathrm{C} 2$} & Universitarias & & $Z=2,93 ; p=0,02$ & $\mathrm{Z}=\mathbf{5 , 7 1 ;} \mathbf{p}<\mathbf{0 , 0 0 1}$ & $Z=6,39 ; p<0,001$ \\
\hline & Universitarios & $\mathrm{Z}=2,93 ; p=0,02$ & & $Z=1,74 ; p=0,4$ & $Z=3,14 ; p=0,009$ \\
\hline & Jubiladas & $Z=5,71 ; p<0,001$ & $Z=1,74 ; p=0,4$ & & $Z=1,81 ; p=0,4$ \\
\hline & Jubilados & $\mathrm{Z}=\mathbf{6 , 3 9 ;} \mathbf{p}<\mathbf{0 , 0 0 1}$ & $Z=3,14 ; p=0,009$ & $Z=1,81 ; p=0,4$ & \\
\hline \multirow[t]{4}{*}{$\mathrm{C} 3$} & Universitarias & & $\mathrm{Z}=2,01 ; \mathrm{p}=0,2$ & $\mathrm{Z}=\mathbf{6 , 8 5} ; \mathbf{p}<0,001$ & $\mathrm{Z}=\mathbf{5 , 5 2 ; p < 0 , 0 0 1}$ \\
\hline & Universitarios & $\mathrm{Z}=2,01 ; \mathrm{p}=0,2$ & & $\mathrm{Z}=\mathbf{3 , 4} ; \mathbf{p}=\mathbf{0 , 0 0 3}$ & $Z=3,09 ; p=0,011$ \\
\hline & Jubiladas & $Z=6,85 ; p<0,001$ & $\mathrm{Z}=\mathbf{3 , 4} ; \mathrm{p}=\mathbf{0 , 0 0 3}$ & & $\mathrm{Z}=0,23 ; \mathrm{p}=1$ \\
\hline & Jubilados & $\mathrm{Z}=5,52 ; \mathrm{p}<0,001$ & $Z=3,09 ; p=0,011$ & $\mathrm{Z}=0,23 ; \mathrm{p}=1$ & \\
\hline \multirow[t]{4}{*}{$\mathrm{C} 4$} & Universitarias & & $Z=3,06 ; p=0,013$ & $Z=6,22 ; p<0,001$ & $\mathrm{Z}=6,11 ; p<0,001$ \\
\hline & Universitarios & $Z=3,06 ; p=0,013$ & & $Z=2,01 ; p=0,2$ & $Z=\mathbf{Z}, \mathbf{8 1} ; p=\mathbf{0 , 0 2 9}$ \\
\hline & Jubiladas & $Z=6,22 ; p<0,001$ & $\mathrm{Z}=2,01 ; \mathrm{p}=0,2$ & & $\mathrm{Z}=1,20 ; \mathrm{p}=1$ \\
\hline & Jubilados & $Z=6,11 ; p<0,001$ & $Z=2,81 ; p=0,029$ & $Z=1,20 ; p=1$ & \\
\hline
\end{tabular}

Los resultados mostrados en la prueba de Kruskal Wallis para la actitud hacia el bienestar animal en general (C-Total) se pueden corroborar en la figura 1, en la que se muestran tanto la mediana como las respuestas que presentan una frecuencia entre el 25 y el $75 \%$, así como los valores máximo y mínimo de respuesta. Como puede comprobarse, los grupos más parecidos son jubiladas y jubilados, de hecho, no se muestran significativamente diferentes. Otro aspecto de interés visible en la gráfica es que el mayor rango de respuesta lo muestra el grupo universitarios y el menor, el grupo universitarias. Efectivamente, dicho grupo es el que presenta una mayor concentración de los valores que suponen el $25-75 \%$ de respuestas. Es decir, la respuesta del grupo universitarias es la más homogénea. 
Figura 1. Medianas de C-Total de los 4 grupos estudiados

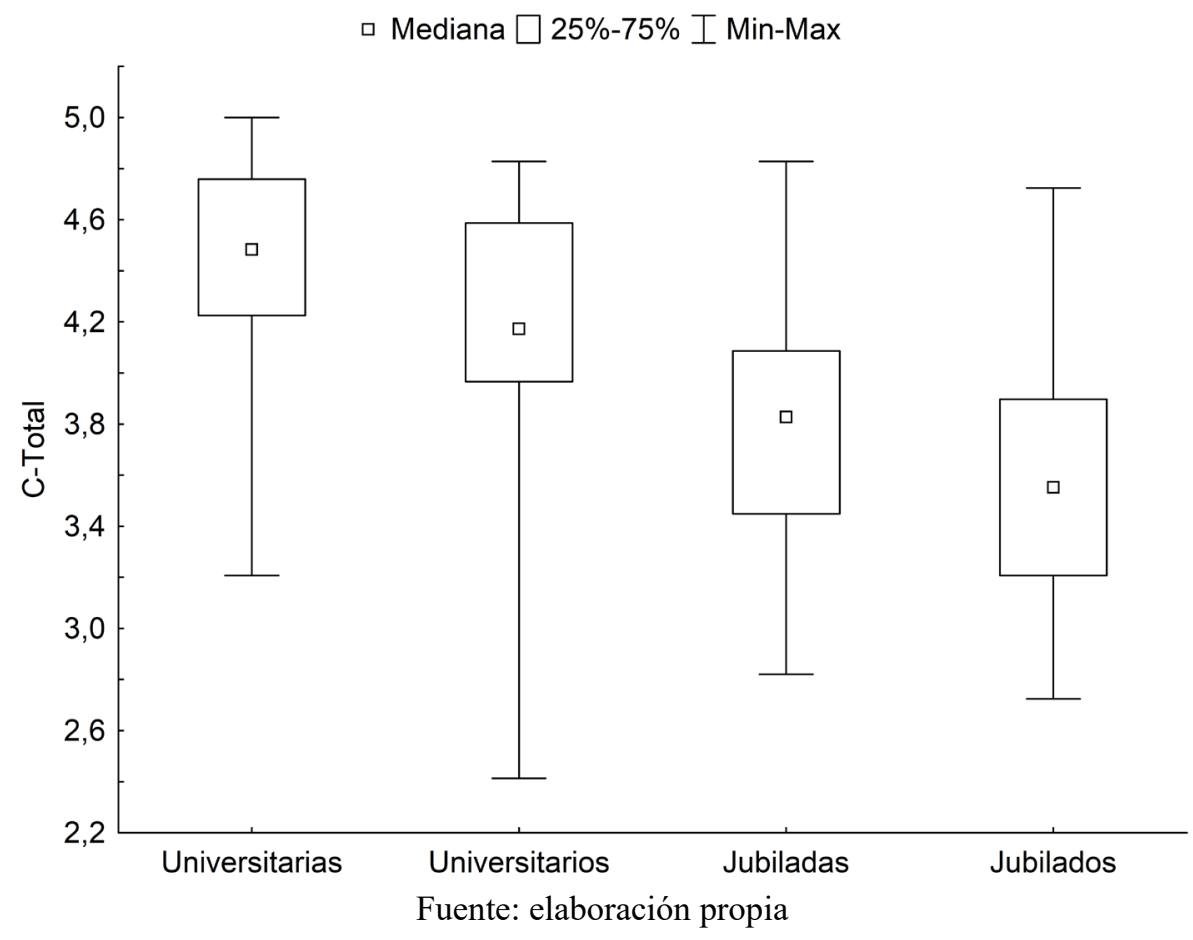

De las otras variables registradas: localidad y tener mascotas, no hay diferencias significativas en localidad (pueblo o ciudad; $U$ de Mann-Whitney $Z=-0,73$; $p=0,4$ ), pero sí entre los que tienen mascota y los que no la tienen ( $U$ de Mann-Whitney $Z=5,40 ; p<0,001$ ) en el componente general CTotal. Si comparamos dentro de cada grupo si existen diferencias entre los que tienen mascota y los que no, encontramos diferencias significativas dentro de todos los grupos (Universitarias: $Z=3,42$; $p<0,001$; Universitarios: $Z=2,50 ; p=0,012$; Jubiladas: $Z=2,60 ; p=0,009$; Jubilados: $Z=2,54 ; p=0,01$ ). Aunque en los tres primeros grupos los valores son mayores en el caso de tener mascota, menos en el grupo de jubilados que es mayor en los que no tienen mascota (Tabla 5).

TABLA 5. Puntuación media (y medianas) de actitud hacia el bienestar animal (C-Total) por grupo y género respecto a tener o no tener mascota

\begin{tabular}{lrrrr}
\hline & Universitarias & Universitarios & Jubiladas & Jubilados \\
\hline Tengo mascota & $4,51(4,60)$ & $4,30(4,44)$ & $4,06(4,03)$ & $3,29(3,24)$ \\
No tengo mascota & $4,31(4,36)$ & $3,90(4,06)$ & $3,71(3,67)$ & $3,75(3,67)$ \\
\hline
\end{tabular}

Fuente: elaboración propia

\section{DiSCUSIÓN Y CONCLUSIONES}

De los resultados obtenidos y analizados en el presente estudio, podemos concluir que la valoración global de todas las personas participantes muestra que su actitud hacia el bienestar animal en general es favorable, con resultados de sus medias superiores a los 3 puntos y próximos a los 4 puntos (en una escala de 5 puntos), o lo que es lo mismo, "estar de acuerdo" con las afirmaciones favorables hacia el bienestar animal y rechazando las desfavorables. 
Respecto a la valoración por género, los resultados muestran una actitud más favorable hacia el bienestar animal en todas las dimensiones que recoge la investigación, del género femenino respecto al masculino (dentro de cada grupo de pertenencia), o lo que es lo mismo, las mujeres universitarias tienen mejor puntuación que los hombres, e igual ocurre con las personas mayores de sesenta años jubiladas, donde las mujeres tienen puntuaciones más altas que los hombres, es decir, actitudes más favorables. Sin embargo, solo se muestran diferencias significativas en relación con el género entre estudiantes de universidad. En concreto, las diferencias se muestran significativas en cuanto a la actitud hacia el bienestar animal en general (C-Total) y en cuanto al maltrato por placer o por desconocimiento $(\mathrm{C} 1)$. En otros estudios se ha mostrado cómo las mujeres poseen una actitud más favorable hacia el bienestar animal que los hombres (Herzog, 2007; Phillips et al., 2011), lo que puede ser debido a una mayor empatía hacia otras personas y hacia los animales (Taylor y Signal, 2005).

Por otro lado, hemos comprobado cómo la pertenencia a un grupo de edad es también un factor determinante, pudiendo afirmar que el alumnado de universidad tiene una actitud más favorable respecto al bienestar animal que las personas mayores de sesenta años jubiladas. Ante lo expuesto se confirma que las mujeres universitarias tienen las puntuaciones más altas de actitud hacia el bienestar animal, seguidas de los hombres universitarios, y tras estos irían en primer lugar las mujeres mayores de sesenta años jubiladas y en último lugar en con las puntuaciones más bajas, y por tanto una actitud menos favorable hacia el bienestar animal, los hombres mayores de sesenta años jubilados. En un estudio centrado en la percepción pública del bienestar de los animales de granja en España mostraba mayor sensibilidad hacia el bienestar en los jóvenes, las mujeres y estudiantes (María, 2006), lo que coincidiría con los resultados aquí mostrados.

Sin embargo, los resultados de los análisis ponen de manifiesto que no hay diferencias significativas entre el grupo universitarios y el grupo jubiladas en la actitud hacia el maltrato animal por placer o por desconocimiento (C1), ni en el componente de ocio con animales (C2), ni en el de abandono de animales (C4). En el caso de universitarios y jubilados, no es diferente significativamente del componente $\mathrm{C}$ 1. La conclusión que se obtiene es que el análisis de dos grupos de pertenencia aparentemente muy distales como son el alumnado universitario y las personas mayores de 60 años jubiladas resulta de interés al entrar en detalle, puesto que las divergencias no son tan elevadas como cabría esperar, mostrándose actitudes intermedias como las protagonizadas por los grupos de universitarios y jubiladas.

La dimensión referente al ocio con animales (C2) es la peor valorada por los participantes en el estudio, como podemos observar en los datos de la tabla 3 (puntuación media de actitud hacia el bienestar animal por dimensiones), siendo los ítems de la misma los que obtienen menores puntuaciones. Igual resultado se obtiene en la comparativa con los datos resultantes del estudio de Mazas et al. (2013) en el que las puntuaciones de los componentes se pueden ordenar de forma similar. Se piensa que se debe a la tradición cultural arraigada en España visible para todos los grupos de edad de nuestra sociedad, con espectáculos de ocio con animales como encierros de vaquillas, corridas de toros, la caza en sus diferentes expresiones, espectáculos circenses y demás actividades o espectáculos con animales, que hace que las personas tengan una actitud hacia el bienestar animal de esta dimensión menos favorable que en el resto de dimensiones estudiadas.

También coinciden nuestros resultados con los obtenidos por Mazas et al. (2013) en cuanto a la dimensión mejor estimada, siendo la referente al " $\mathrm{C} 1$ : maltrato por placer y por desconocimiento". Esta obtuvo la puntuación media más alta en todos los grupos de estudio, comparándola con las puntuaciones medias del resto de componentes y de la actitud hacia el bienestar animal general. Esta mayor puntuación podría atribuirse a que uno de los conceptos de maltrato animal más aceptado por los españoles es el que lo relaciona con aquellos comportamientos de las personas que causan un daño innecesario a los animales, provocados tanto de forma activa o voluntaria, como de forma pasiva, cuando es resultado de alguna negligencia o falta de atenciones necesarias para su salud (Fernández, 2019). Una variable que se ha considerado 
en este estudio es la tenencia de una mascota, que influye de forma favorable en la actitud hacia el bienestar animal en todos los grupos menos en el de hombres jubilados, que es el único grupo con una tendencia opuesta, lo que puede indicar una concepción distinta del bienestar animal. En otros grupos de edad en los que se ha estudiado la influencia de tener una mascota, por ejemplo en niños, se ha visto que los que poseen mascotas muestran una mejor actitud hacia el bienestar animal (Almedia y García-Fernández, 2020; Menor-Campos, Hawkins y Williams, 2019). Esto es importante desde el punto de vista docente, ya que, para diseñar actuaciones con el objetivo de fomentar mejoras en la actitud hacia el bienestar animal, estas pueden contemplar la presencia de mascotas, que es un tipo de actividad ampliamente difundida por los beneficios que reporta al alumnado (Brelsford, Meints, Gee y Pfeffer, 2017).

La constatación de una actitud favorable hacia el bienestar animal es particularmente relevante porque el alumnado participante cursa los Grados de Educación Primaria e Infantil y en un futuro cercano tendrán la responsabilidad de ejercer su profesión en las aulas. Por otro lado, se ha de poner en valor la actitud hacia el bienestar animal de las mujeres universitarias, que suelen poseer un comportamiento más proambiental que los hombres (Casaló y Escario, 2018), aunque el debate del porqué de estas diferencias de género es complejo y podría deberse a la teoría de la socialización de género (McCright y Xiao, 2014; Strapko, Hempel, MacIlroy y Smith, 2016). Las diferencias con el resto de los grupos nos deben llevar a analizar en futuros trabajos las claves que las sustentan, para potenciar dichos aspectos en la educación para el bienestar animal en las aulas.

A la vista de los resultados favorables del grupo de maestras y maestros en formación, una recomendación sería que no es necesario trabajar en los grados de educación con la intención de fomentar o incrementar las actitudes favorables hacia el bienestar animal y que sería suficiente si se tratara de forma transversal al trabajar aspectos de los animales, aunque sí sería recomendable que conociesen este término.

Por otra parte, llama la atención que en una sociedad con abundancia de mascotas no se aborde explícitamente el término bienestar animal en los libros de texto, cuando sí ha llamado la atención de la sociedad, con numerosos grupos activistas que han realizado campañas y acciones para divulgar su mensaje (Díaz Carmona, 2012), aunque sí se exponen en dichos libros actividades y experiencias dirigidas hacia la protección de los animales (Mazas y Fernández Manzanal, 2016). Aún así los datos indican que es en secundaría cuando los estudiantes muestran actitudes menos favorables (Mazas et al., 2013) y es en esta etapa entonces donde se tendría que hacer un mayor esfuerzo por abordar este tema.

Este estudio muestra a su vez la actitud hacia el bienestar animal en un grupo escasamente estudiado, como son las personas mayores de 60 años, pero que siguen recibiendo formación mediante actividades y talleres que se desarrollan en la programación de los Centros de Participación Activa (CPA) o en las aulas universitarias para mayores y que, por lo tanto, pueden tener en consideración los resultados aquí obtenidos para trabajar el bienestar animal en este grupo de edad, igual que se ha recomendado que se trabaje en estudiantes (Mazas y Fernández Manzanal, 2016).

A pesar de las actitudes favorables de la mayoría de la población estudiada, el número de delitos por maltrato animal sigue siendo importante y se incrementa cada año, aunque parece que las causas pueden no ser un aumento de maltratos, sino una sensibilización y concienciación de la ciudadanía para denunciar los hechos (Gavilán Rubio, 2017), lo que podría mostrar la importancia de fomentar actitudes favorables hacia el bienestar animal y que las actitudes generales de la población son bastante favorables.

La educación ambiental debe tener cada vez más importancia en la educación y en los distintos currículos debido a la crisis ambiental global a la que se enfrenta la humanidad, por lo que el fomento de una actitud favorable hacia el bienestar animal podría complementar las actitudes hacia el medioambiente, respeto de la naturaleza y de los seres vivos. Es importante destacar que los temas y problemáticas medioambientales son muy complejos y controvertidos, y que los 
educadores buscan involucrar a las personas en los desafíos de la sostenibilidad, pero tradicionalmente la educación científica se ha centrado más en la enseñanza de conocimientos y habilidades, mientras que la educación medioambiental enfatiza la incorporación de valores y el cambio de comportamientos. Según Wals, Brody, Dillon y Stevenson (2014) se debe fomentar la convergencia de la educación científica y la educación ambiental, lo que podría involucrar a las personas en abordar desafíos socioecológicos fundamentales.

No podemos dejar de mencionar las limitaciones de este trabajo, que aunque siendo la primera vez que se muestran las actitudes del sector de la población mayor de 60 años, no estudia la población intermedia entre estudiantes y personas jubiladas, es decir, un sector numeroso y muy importante de la sociedad, por lo que se postula como línea futura de investigación.

\section{Referencias}

Ajzen, I. (2001). Nature and operation of attitudes. Annual Review of Psychology, 52, 27-58. DOI: 10.1146/annurev.psych.52.1.27

Ajzen, I. (2005). Attitudes, personality, and behavior. McGraw-Hill Education (UK).

Ajzen, I. y Fishbein, M. (1977). Attitude-behavior relations: A theoretical analysis and review of empirical research. Psychological bulletin, 84(5), 888.

Ajzen, I. y Fishbein, M. (2000). Attitudes and the attitude-behaviour relation: Reasoned and automatic processes. En W. Stroebe y M. Hewstone (Eds.), European review of social psychology (pp. 1-33). Chichester: Wiley.

Almeida, A. y García Fernández, B. (2020). Attitudes towards animal welfare in Portuguese students from the 6th and the 9th year of schooling: implications for environmental education. Environmental Education Research, 1-25. DOI: 10.1080/13504622.2020.1858028

Ascione, F. R. (2015). Children who are cruel to animals: A review of research and implications for developmental psychopathology. Anthrozoös, 6, 226-247. DOI: $\underline{10.2752 / 089279393787002105}$

Bentham, J. (1789). The principles of Morals and Legislation. Londres: Payne. Republished, Oxford: Clarendon Press.

Bisquerra, R. (2000). Metodología de la investigación educativa. Madrid: La Muralla.

Brambell, R. (1965). Report of the technical committee to enquire into the welfare of animals kept under intensive livestock husbandry systems. Londres: Her Majesty's Stationary Office.

Brelsford, V. L., Meints, K., Gee, N. R. y Pfeffer, K. (2017). Animal-Assisted Interventions in the Classroom - a systematic review. International journal of environmental research and public health, 14(7), 669. DOI: 10.3390/ijerph14070669

Broida, J., Tingley, L., Kimball, R. y Miele, J. (1993). Personality differences between Pro- and Anti-vivisectionists. Society \& Animals 1(2), 129-144. DOI: $\underline{10.1163 / 156853093 X 00037}$

Campo-Arias, A. y Oviedo, H. C. (2008). Propiedades psicométricas de una escala: la consistencia interna. Revista de salud pública, 10, 831-839.

Casaló, L. V. y Escario, J. J. (2018). Heterogeneity in the association between environmental attitudes and pro-environmental behavior: A multilevel regression approach. Journal of Cleaner Production, 175, 155-163. DOI: 10.1016/j.jclepro.2017.11.237

COM (2012-2015). Comunicación de la Comisión al Parlamento Europeo, al Consejo y al Comité Económico y Social Europeo relativa a la estrategia de la Unión Europea para la protección y el bienestar de los animales 2012-2015.

Dawkins, M. S. (2004). Using behaviour to assess welfare. Animal Welfare, 13, S3-S7.

Díaz Carmona, E. (2012). Perfil del vegano/a activista de liberación animal en España. Revista Española de Investigaciones Sociológicas (REIS), 139(1), 175-187.

Fernández-Álvarez, A. (2019). Elementos básicos del maltrato animal. Amazing Books. Zaragoza. España. 
Fernie, A. C., Tribe, A., Murray, P. J., Lisle, A. y Phillips, C. J. C. (2012). A survey of the attitudes of stakeholders in the zoo industry towards the husbandry requirements of captive Great Apes. Animal Welfare-The UFAW Journal, 21(2), 233.

García Menacho, V. y García Romero, C. (2012). Avicultura ecológica de puesta. Madrid: Ed. Agrícola España.

Gavilán Rubio, M. (2017). El delito de maltrato animal. Sus penas y ejecución de las mismas. Medidas de protección animal en el proceso penal. Anuario Jurídico y Económico Escurialense, (50), 143-166.

George, K. A., Slagle, K. M., Wilson, R. S., Moeller, S. J. y Bruskotter, J. T. (2016). Changes in attitudes toward animals in the United States from 1978 to 2014. Biological Conservation, 201, 237-242. DOI: 10.1016/j.biocon.2016.07.013

Gutiérrez, G., Granados, D. R. y Piar, N. (2007). Interacciones humano-animal: características e implicaciones para el bienestar de los humanos. Revista Colombiana de Psicología, 16, 163184.

Henry, B. C. (2004). The relationship between animal cruelty, delinquency, and attitudes toward the treatment of animals. Society and Animals, 12(3), 185-207. DOI: $\underline{10.1163 / 1568530042880677}$

Herzog, H., Betchart, N. y Pittman, R. (1991). Sex role identity and attitudes toward animals. Anthrozoös 4(3), 184-192.

Herzog, H. A. (2007). Gender differences in human-animal interactions: A review. Anthrozoös, 20(1), 7-21. DOI: $\underline{10.2752 / 089279307780216687}$

Herzog, H. A. y Golden, L. L. (2009). Moral emotions and social activism: The case of animal rights. Journal of Social Issues, 65(3), 485-498. DOI: 10.1111/j.1540-4560.2009.01610.x

Hills, A. M. (1995). The motivational bases of attitudes toward animals. Society \& Animals, 1, 111128.

Horgan, R. y Gavinelli, A. (2006). The expanding role of animal welfare within EU legislation and beyond. Livestock Science, 103(3), 303-307. DOI: 10.1016/i.livsci.2006.05.019

Jamieson, J., Reiss, M., Allen, D., Asher, L., Wathes, C. y Abeyesinghe, S. (2012). Measuring the success of a farm animal welfare education event. Animal Welfare, 21, 65-75. DOI: $\underline{10.7120 / 096272812799129402}$

Kaiser, H. F. (1974). An index of factorial simplicity. Psychometrika, 39, 31-36.

Kellert, S. R. (1988). Human-animal interactions: A review of American attitudes toward wild and domestic animals in the 20th century. In Animals and People Sharing the World, 137-175, ed. A. N. Rowan. Hanover, NH: University Press of New England.

López, M. T. (2005). Derechos de los animales, deberes de los humanos. Universidad de Salamanca. España. Isegoría $\mathrm{N}^{\mathrm{o}} 32$.

Lundmark, F., Berg, C., Schmid, O., Behdadi, D. y Röcklinsberg, H. (2014). Intentions and values in animal welfare legislation and standards. Journal of Agricultural and Environmental Ethics, 27(6), 991-1017. DOI: 10.1007/s10806-014-9512-0

María, G. A. (2006). Percepción pública del bienestar de los animales de granja en España. Ciencia del ganado, 103, 250-256.

Mathews, S. y Herzog, H. (1997). Personality and attitudes towards the treatment of animals. Society \& Animals, 5(2), 57-63.

Mazas, B. y Fernández Manzanal, R. (2016). El concepto de bienestar animal en el currículo de Secundaria y en los libros de texto de ciencias. Revista Eureka sobre Enseñanza y Divulgación de las Ciencias, 13(2), 301-314. Recuperado de http://hdl.handle.net/10498/18290

Mazas, B. y Fernández Manzanal, R. F. (2018a). Desarrollo de una escala de actitudes hacia el bienestar animal para estudiantes de Educación Primaria. Revista Complutense de Educación, 29(4), 33-52. DOI: $\underline{10.5209 / R C E D .54883}$ 
Mazas, B. y Fernández-Manzanal, R. (2018b). A more fine-grained measure towards animal welfare: a study with regards to gender differences in Spanish students. Environmental Science and Pollution Research, 26(1), 844-854. DOI: 10.1007/s11356-018-3537-2

Mazas, B., Fernández Manzanal, M. R., Zarza, F. J. y María, G. A. (2013). Development and validation of a scale to assess students' attitude towards animal welfare. International Journal of Science Education, 35(11), 1775-1799. DOI: 10.1080/09500693.2013.810354

McCright, A. M. y Xiao, C. (2014). Gender and environmental concern: Insights from recent work and for future research. Society \& Natural Resources, 27(10), 1109-1113. DOI: 10.1080/08941920.2014.918235

McCulloch, S. P. (2013). A critique of FAWC's five freedoms as a framework for the analysis of animal welfare. Journal of agricultural and environmental ethics, 26(5), 959-975.

Melik, N. (2007). Francia y los derechos de los animales. En: Bienestar animal (pp.211-224). Zaragoza: Ed. Acribia.

Mellor, D. J. (2016). Updating animal welfare thinking: Moving beyond the "Five Freedoms" towards "a Life Worth Living". Animals, 6(3), 21. DOI: 10.3390/ani6030021

Mendl, M., Burman, O. H., Parker, R. M. y Paul, E. S. (2009). Cognitive bias as an indicator of animal emotion and welfare: Emerging evidence and underlying mechanisms. Applied Animal Behaviour Science, 118(3-4), 161-181. DOI: 10.1016/j.applanim.2009.02.023

Menor-Campos, D. J., Diverio, S., Sánchez-Muñoz, C., López-Rodríguez, R., Gazzano, A., Palandri, L. y Mariti, C. (2019). Attitudes toward animals of students at three European veterinary medicine schools in Italy and Spain. Anthrozoös, 32(3), 375-385. DOI: $\underline{10.1080 / 08927936.2019 .1598658}$

Menor-Campos, D. J., Hawkins, R. y Williams, J. M. (2019). Attitudes Toward Animals Among Spanish Primary School Children. Anthrozoös, 32(6), 797-812. DOI: 10.1080/08927936.2019.1673055

Miranda-de la Lama, G. C., Pascual-Alonso, M., Guerrero, A., Alberti, P., Alierta, S., Sans, P., Gajan, J. P., Villarroel, M., Dalmau, A., Velarde, A., Campo, M. M., Galindo, F., Santolaria, M. P., Sañudo, C. y María, G. A. (2013). Influence of social dominance on production, welfare and the quality of meat from beef bulls. Meat Science, 94, 432-437. DOI: 10.1016/j.meatsci.2013.03.026

Mosterín, J. (1998). ¡Vivan los animales! Madrid: Ed. Debate.

Paul, E. S. y Serpell, J. A. (1993). Childhood pet keeping and humane attitudes in young adulthood. Animal Welfare 2(4), 321-337.

Phillips C. J. C., Izmirli S., Aldavood S. J., Alonso M., Choe B. I., Hanlon A., Handziska, A., Illmann, G., Keeling, L., Kennedy, M., Lee, G. H., Lund, V., Mejdell, C., Pelagic, V. R. y Rehn, T. (2011). An International Comparison of Female and Male Students' Attitudes to the Use of Animals. Animals, 1, 7-26. DOI: 10.3390/ani1010007

Phillips, C. J. C., Izmirli S., Aldavood, S. J., Alonso, M., Choe, B. I., Hanlon, A., Handziska, A., Illmann, G., Keeling, L., Kennedy, M., Lee, G. H., Lund, V., Mejdell, C., Pelagic, V. R. y Rehn, T. (2012). Students' attitudes to animal welfare and rights in Europe and Asia. Animal Welfare, 21(1), 87-100.

Prokop, P. y Randler, C. (2018). Biological predispositions and individual differences in human attitudes toward animals. In Ethnozoology (pp. 447-466). Academic Press.

Serpell, J. (2000). The domestic dog. Cambridge: Cambridge University Press.

Spooner, J. M., Schuppli, C. A. y Fraser, D. (2012). Attitudes of Canadian beef producers toward animal welfare. Animal Welfare, 21(2), 273-283

Spooner, J. M., Schuppli, C. A. y Fraser, D. (2014). Attitudes of Canadian citizens toward farm animal welfare: A qualitative study. Livestock Science, 163, 150-158. DOI: $\underline{10.1016 / j . l i v s c i .2014 .02 .011}$ 
Strapko, N., Hempel, L., MacIlroy, K. y Smith, K. (2016). Gender differences in environmental concern: Reevaluating gender socialization. Society \& natural resources, 29(9), 1015-1031. DOI: $10.1080 / 08941920.2016 .1138563$

Taylor, N. y Signal, T. D. (2005). Empathy and attitudes to animals. Anthrozoös, 18(1), 18-27. DOI: $10.2752 / 089279305785594342$

Vallejo, P. M. (2006). Medición de actitudes en psicología y educación: construcción de escalas y problemas metodológicos (Vol. 80). Universidad Pontifica Comillas.

Ventura-León, J. L. y Caycho-Rodríguez, T. (2017). El coeficiente Omega: un método alternativo para la estimación de la confiabilidad. Revista Latinoamericana de Ciencias Sociales, Niñez y Juventud, 15(1), 625-627.

Wals, A. E. J., Brody, M., Dillon, J. y Stevenson, R. B. (2014). Convergence between science and environmental education. Science, 344(6184), 583-584.

Webster, A. J. (2001). Farm animal welfare: the five freedoms and the free market. The veterinary journal, 161(3), 229-237. DOI: $10.1053 /$ tvj1.2000.0563

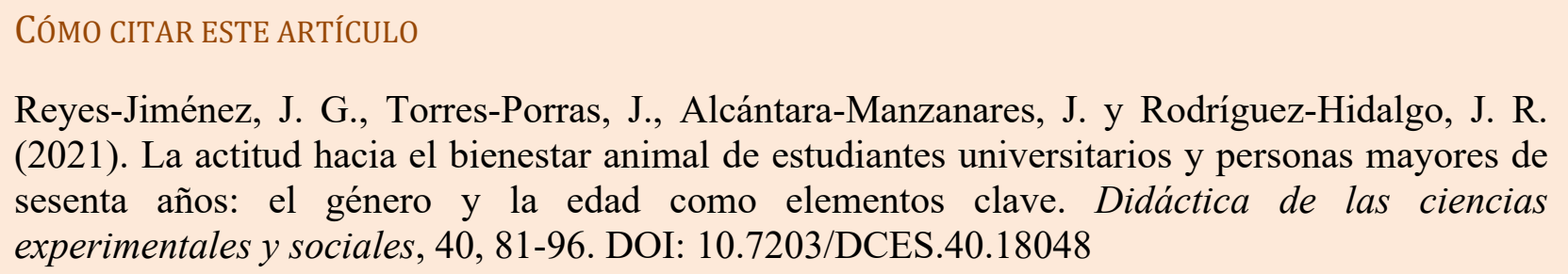

Reyes-Jiménez, J. G., Torres-Porras, J., Alcántara-Manzanares, J. y Rodríguez-Hidalgo, J. R. (2021). La actitud hacia el bienestar animal de estudiantes universitarios y personas mayores de sesenta años: el género y la edad como elementos clave. Didáctica de las ciencias experimentales y sociales, 40, 81-96. DOI: 10.7203/DCES.40.18048 
